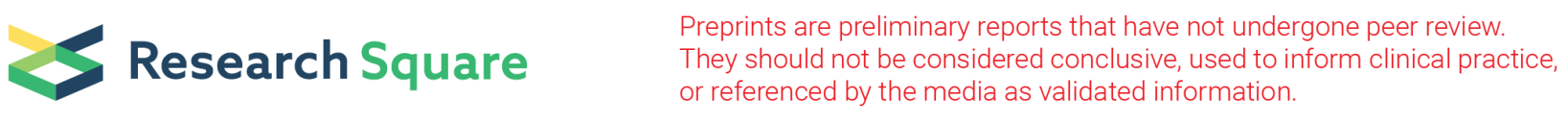

\title{
Psychosocial supports utilized by caregivers of patients with dementia in South western Uganda: A qualitative investigation
}

Catherine Abaasa ( $\sim$ acatherine@must.ac.ug )

Mbarara University of Science and Technology(MUST) https://orcid.org/0000-0001-6882-1831

Celestino Obua

Mbarara University of Science and Technology

Edith K Wakida

Mbarara University of Science and Technology

Godfrey Zari Rukundo

Mbarara University of Science and Technology

Research article

Keywords: Alzheimer's disease, dementia, LMIC, psychosocial support, caregiver

Posted Date: September 17th, 2019

DOI: https://doi.org/10.21203/rs.2.14529/v1

License: (c) (i) This work is licensed under a Creative Commons Attribution 4.0 International License.

Read Full License 


\section{Abstract}

Background Patients with dementia experience problems ranging from mild to severe impairments in daily life activities. In Uganda, like in most other low and middle-income countries, families are a cornerstone in providing care to patients with dementia. Serving as a caregiver for a person with Alzheimer's disease is a physically, emotionally and financially demanding role, that is associated with increased stress, depression, fatigue, and financial strain. These have often resulted in elder abuse or neglect of the patients. Currently, data on psychosocial supports available to caregivers of patients with dementia in Uganda is not available.

Methods We conducted a qualitative assessment of the psychosocial supports available to caregivers of patients with Alzheimer's disease and related dementias in southwestern Uganda, including the content of the formal and informal support interventions and by whom they are delivered.

Results Medical practitioners provide educational support on the diagnosis and prognosis of dementia, but may not provide emotional support in the form of counseling to caregivers of patients with dementia. Caregivers in Southwestern Uganda, receive unstructured emotional and instrumental support from sources outside of the medical system such as family and community, which leave the Caregivers in confusion.

Conclusions The medical care system offers some information about the clinical presentation of Alzheimer's disease but little or no psychosocial support to the Caregivers of patients with the disease. This has implications for how families and Caregivers cope with the day-to-day care of patients with Alzheimer's disease in Uganda and calls for institutionalization of structured psychosocial support for Caregivers to improve the care given to Alzheimer's patients.

\section{Background}

Dementia is a clinical syndrome characterized neurodegeneration that causes irreversible decline in cognitive functioning. The most common cause of dementia is Alzheimer's Disease (1). More than 35 million people over the age of 60 are living with dementia worldwide and $62 \%$ of them resides in low and middle-income countries (LMICs), and is expected to rise to $66 \%$ by 2030 (2). In sub-Saharan Africa, 2.13 million people experience dementia, including $4.5 \%$ of individuals over the age of 60 in East sub-Saharan Africa (3). As this prevalence is expected to rise 65.7 million by 2030, this rapid growth in prevalence will place higher demands on dementia care in this region(4).

Individuals with Alzheimer's disease and related dementias (ADRD) experience impairments in memory, communication, attention, reasoning, judgment, and visual perception as well as changes in 
mood and personality that limit their ability to function independently(5). The behavioral and neuropsychiatric problems associated with dementia (e.g., anger, worry, wandering and delusions) in combination with the affected individual's daily care needs (feeding, dressing, bathing) place a high burden on informal caregivers, such as family members, friends, paid in-home assistance (6-8). Accordingly, these caregivers experience high level of chronic stress and depressive and anxiety disorders, which can in turn exacerbate the behavioral and psychological symptoms of dementia (9-12). Therefore, the implementation of effective ADRD caregiver support interventions is central to reducing caregiver burden and behavioral symptoms among patients.

To date, the vast majority of this research on caregiver burden in ADRD has been conducted in high-income countries. An emerging body of research from LMICs suggests that informal caregivers in LMICs also experience emotional distress associated with managing their loved one's daily care routines and challenging behavioral symptoms (13-15). Caregiver burden and associated distress in LMICs is also compounded by lack of social support and shortage of formal specialized medical care for people with ADRD (16-19). Given the projected rapid increase in ADRD prevalence in sub-Saharan Africa, enhancing support for caregivers of persons with ADRD in this region is of urgent importance (8).

In high income-countries interventions for caregivers of people with ADRD that target enhancing caregivers' knowledge about the disease, and skills for managing challenging behavioral symptoms have been shown to reduce caregiver depression and improve patient neuropsychiatric symptoms $(20,21)$. Similarly interventions targeting caregiver dementia knowledge and behavior management skills have been shown to reduce skills, reduce caregiver burden and distress in India and Russia $(22,23)$. However, there is a paucity of data on caregiver interventions in sub-Saharan Africa. A recent review of qualitative studies from sub-Saharan Africa, including studies from South Africa, Tanzania, Nigeria, Ghana, and Congo revealed that caregiving for people with dementia is a collective responsibility among family members and that families often utilized biomedical and non-biomedical treatment approaches such as traditional healers $(24,25)$. Caregivers also descried a desire for further education on dementia from the treating medical providers, and expressed need for greater emotional and financial support to meet their family member's needs (3). 
To date, there are no data available on ADRD caregiver support interventions in Uganda. Given the limited availability of specialty mental health care in Uganda, it is essential to investigate what formal and informal support services are currently being utilized by caregivers of people with ADRD. Understanding the support interventions currently utilized in rural Uganda will inform the adaptation and integration of evidence-based caregiver support interventions in ADRD care in this setting. Therefore, this study aimed to characterize the formal and informal supports utilized by caregivers of patients with dementia in southwestern Uganda, including by whom the support is provided and the content of the formal and informal support provided.

\section{Methods}

\section{Study setting and sampling}

Participants were recruited from Mbarara Regional Referral Hospital (MRRH), Kabale Regional Referral Hospitals (KRRH), and Kampala International Teaching Hospital (KIUTH) in Southwestern Uganda. These institutions offer specialized Psychiatric services to Southwestern Uganda, together serving a catchment of $8,874,862$ people, $14 \%$ of which are over 60 years of age (26). Though caregiver support for dementia is delivered locally within Parish-based health centers, participants were recruited from the regional referral hospitals to leverage the diagnostic expertise of psychiatric staff at the regional referral hospitals for the identification of dementia.

Caregivers of people with dementia were purposively sampled from the outpatient psychiatry clinics of MRRH, KRRH and KIUTH. Due to methodological limitations at these sites, a clinical diagnosis of Alzheimer's disease it not made at these sites. Patients are instead diagnosed with dementia, which is classified as HIV-related or non-HIV-related. Non-HIV-related dementia is considered to be possible ADRD. The caregivers of the patients diagnosed with dementia were identified by the treating clinician and then approached by study staff who introduced the study and explained the study purpose and objectives. Upon expressing interest in the study, study staff scheduled an appointment to obtain informed consent and complete the interview at the convenience of the caregiver. Participants were eligible if they were: 1) caring for someone with a diagnosis of dementia aged 60 years and above, 2) had taken care of the person for more than six months, 3) self-identified as the primary caregiver, 4) was a family member or friend of the patient, and 5) was 18 years and above. Caregivers of people with HIV-related dementia were 
excluded. Written informed consent was obtained from each participant. Participants who could not read and write documented their consent with a thumbprint in the presence of a witness. The interviews with the caregivers were conducted at a private location, often in their homes, and at a time convenient for the caregiver.

\section{Interview procedures}

An in-depth interview guide was developed to explore the dementia caregiver support services available in rural southwestern Uganda, including the type of services utilized by caregivers and by whom the services are provided. The questions included (a) what kind of counseling services is available for you as a care-giver of a patient with Alzheimer's disease in South Western Uganda? (b) Who usually gives the counseling? What is done during counseling? What do you think of these activities? (c) What kind of information is provided during counseling (d) What other sources of counseling services are available for you as a caregiver of a patient with Alzheimer's disease in Southwestern Uganda? (e) What resources at family and community level do you access that help you to care for the patients with dementia? The interview guides were translated into Runyankore-Rukiga, the local language, and back translated into English to ensure that the message was correctly translated. The interview guides were first piloted with four caregivers of patients with dementia at MRRH and iteratively revised. In-depth interviews were then conducted in Runyankore-Rukiga by the lead author and two trained research assistants between December 2018 and January 2019. Interviews lasted between 30-75 minutes and were audio recorded. Indepth interviews were conducted until thematic saturation was reached.

\section{Data management and analysis}

In-depth interviews were translated from Runyankore-Rukiga into English and transcribed by the research assistants, and checked by CA against the audio recordings for correctness of information. Thematic content analysis was used to analyze the data (27). CA reviewed transcripts and audio recordings, and developed a codebook based on emerging themes. CA and ESO double coded $29 \%$ of transcripts compared their findings, and discussed and harmonized their differences until consistency in coding was achieved. The remaining interview transcripts were coded by CA independently. Data analysis was conducted using qualitative data analysis software, ATLAS.ti version 7 (28). The research team (CA, GZR and $\mathrm{CO}$ ) discussed the thematic coding in the codebook, the results of the analysis in Atlas .ti and 
came up with final major themes as caregiver support services and services utilized beyond the medical care system.

\section{Results}

\section{Participant characteristics}

A total of thirty-four interviews were conducted across the three hospitals in southwestern Uganda. Of the caregivers, daughters comprised about one-third (32.4\%); most were married (64.7\%), and slightly over one-third were aged between $44-53$ years (35.3\%). Only 5 caregivers $(14.7 \%)$ had no formal education, while the majority was of Anglican religious faith (61.8\%). Most of caregivers had been caring for the person with dementia for 5 years or more (70.6\%) (see Table 1).

\section{Table 1 Characteristics of study participants}




\begin{tabular}{|c|c|c|c|}
\hline Participant characteristics & & Freq. & $\%$ \\
\hline \multirow[t]{5}{*}{ Age } & $24-33$ & 6 & 17.6 \\
\hline & $34-43$ & 6 & 17.6 \\
\hline & $44-53$ & 12 & 35.3 \\
\hline & $54-63$ & 6 & 17.6 \\
\hline & 64 and above & 4 & 11.8 \\
\hline \multirow[t]{7}{*}{ Relationship to the patient } & Daughter & 11 & 32.4 \\
\hline & Daughter in law & 7 & 20.6 \\
\hline & Son & 7 & 20.6 \\
\hline & Wife & 5 & 14.7 \\
\hline & Grandson & 1 & 2.9 \\
\hline & Niece & 2 & 5.9 \\
\hline & Friend & 1 & 2.9 \\
\hline \multirow[t]{4}{*}{ Marital Status } & Widowed & 2 & 5.9 \\
\hline & Married & 22 & 64.7 \\
\hline & Single & 7 & 20.6 \\
\hline & Divorced/separated & 3 & 8.8 \\
\hline \multirow[t]{4}{*}{ Education Level } & None & 5 & 14.7 \\
\hline & Primary & 9 & 26.5 \\
\hline & Secondary & 10 & 29.4 \\
\hline & Tertiary & 10 & 29.4 \\
\hline \multirow[t]{4}{*}{ Religion } & Anglican & 21 & 61.8 \\
\hline & Catholic & 8 & 23.5 \\
\hline & Islam & 3 & 8.8 \\
\hline & Pentecostal & 2 & 5.9 \\
\hline \multirow[t]{3}{*}{ Duration of the patient with dementia symptoms (years) } & Less than 5 & 10 & 29.4 \\
\hline & $5-10$ & 13 & 38.2 \\
\hline & Above 10 & 11 & 32.4 \\
\hline
\end{tabular}




\section{Caregiver support services}

Dementia caregiver support services in southwestern Uganda were structured into two major themes 1) medical supports utilized, and 2) supports beyond the medical care system. Discussion of medical supports highlighted information support provided by medical providers, as well as the limits of these support services. Discussion of supports beyond the medical care system included themes of emotional and instrumental support provided by religious leaders, the local community, and family members.

Educational support Participants reported receiving guidance from health professionals, who were typically the nurses and physicians from the patient's care. Guidance was often focused on symptoms of the disease and prognosis. Participants were told the importance of treating comorbid conditions, several management modalities and referral for specialized management of dementia. However, when discussing support services provided within the medical care system, the information given did not include reference to emotional support experienced as they care for their family member with dementia.

"The only counseling, I have received, is from the doctors in-charge of psychiatry about the prognosis of the disease" 49 year old female Caregiver.

"It's basically concerning my patient, annoyed, over reactionary, paranoid i.e. how to be patient with her and not being rude. And telling me how patients with dementia behave such as beating people and over reacting. So knowing all that helped me to take good care of her." 47 year old male Caregiver

Limits of medical support Most of the participants reported not receiving emotional guidance on how to manage patients with dementia. They either reported that they did not know where to get counseling from or that such service is nonexistent.

"I don't get any counseling. I don't even know where they are, because there are no established institutions for me to go for counseling. I am lucky that my wife is a counselor. She worked with AIDS information center so when we get time we sit together and talk and we counsel each other... 
besides that, I am also a trained counselor in my course. I did adult and community education because when we were dealing with old people, we would counsel them so I would counsel them" 65 year old male Caregiver.

The participants highlighted the need for formal counseling services within the health system to better prepare caregivers on how to manage people with dementia.

Participants often noted that they did not know about the disease and in the absence of professional guidance turned to non-professional sources such as guidance from community members, family, and the internet.

"Well it would be good if there were systems and counseling services that could prepare caretakers psychologically probably, we don't know much about the disease itself." 47 year old male Caregiver

"Maybe when the doctor told me about the problem, I went to internet and googled. So there is that part concerning how the people of Alzheimer's diseases are nursed. So I got it from the internet through googling because the doctors had told me that there is no treatment. And on internet I found out that when a patient feeds on coconut oil or put it on bread or food it helps. And I tried it and I got it from internet, so that it could help her" 49 year old female Caregiver

\section{Support utilized beyond the medical care system}

Participants reported receiving "emotional support", as well as "instrumental support" to care for their loved one from sources outside of the medical system, including their religious communities, family and other community members.

\section{Emotional support}

Participants described receiving support from religious leaders including pastors, sheikhs, and priests, and members of their religious community. This support typically took the form of emotional support provided through discussions about personal experiences, prayer, songs, and Holy Communion that was noted to improve the subjective mood and well-being of patients and caregivers. The emotional support was in the form of encouragement and advice on how to accept living with people with dementia. 
This support primarily occur during social encounters and religious services as evidenced by the quotes below

"Some people come from church and visit us and we become happy for the whole day, praying and singing and he feels good. And others come to check on [him] and they talk to him... even community members, family members, and other relatives check on us and when he looks at them, he stops thinking a lot" 56 year old female Caregiver.

"Service!!! Taking my patient to church to socialize with the rest, and sharing with others helps us to remain active other than being lonely back home"39 year old female Caregiver

\section{Instrumental support}

The guidance from community members also included instrumental support, such as informational guidance on how to care as well as physical care for a loved one with dementia. However, this support at times contained conflicting advice which was confusing.

"You as a person you can look at things where by one person tells you to go for prayers and another one tells you to see a doctor, then herbalists when you consider what your doctor tells you, you decide on your own which route to follow and sometimes do not know the right or the wrong side to take your patient" 39 year old female caregiver.

Participants also reported receiving instrumental support in the form of food and money from community members and family. They described that they highly valued this support as they were unable to grow their own crops due to time spent caring for their loved one with dementia.

"May be when it's a harvesting season, when a person is coming to check on you, she may bring for you a cup of porridge, beans because sometimes, I can fail to plant anything because of my patient. And I can't even plant a cup of beans or millet. So whoever comes to check on you, she comes with a cup of flour or beans. That's the only help we get from the community"39 year old female Caregiver 
"We call family members and we sit and talk about where to get medicine like selling her piece of land because medicine is expensive, so we talk about the way forward. She has other children but they are in their homes so when we don't have medicines, they try to get it for her." 33 year old female Caregiver

\section{Discussion}

The socio-demographic characteristics of caregivers varied by age, gender, religious affiliations, marital status and relationship to the patient. Participants in this study were widely varied demographically with a diversity of lived experiences in different contexts and duration of caregiving hence one would expect the participants to have enough information on the psychosocial support available for caregivers of Patients with Alzheimer's disease and related dementias in South western Uganda.

Participants in this study were mainly females either daughters, wives or daughters in law. Caregiving of patients with dementia in low and middle income countries is most commonly by middle-aged mothers who are married or cohabiting, have completed some or all of primary school, and are self-employed, farmers, or unskilled laborers similar to the results of this study (29).

In this qualitative study of psychosocial support available to caregivers of patients with dementia in south western Uganda, we found that medical providers often provide diagnostics and prognostic guidance to caregivers. However, caregivers noted an absence of dementia counseling services within the medical system. Many caregivers expressed a need for further guidance on patient care and behavior management strategies. They reported often turning to non-medical sources for this information, as well as emotional and instrumental support.

These findings are consistent with other studies from sub-Saharan Africa finding that caregiver support and patient care for dementia is often obtained outside of the formal medical care system, such as religious and traditional healers and community supports, though caregivers are eager to gain more biomedical guidance (30-32). These findings differ from treatment recommendation from high-income countries, that promote the delivery of dementia caregiver support interventions targeting dementia 
education, behaviour management strategies, communication skills, and caregiver coping skills within the biomedical care system $(20,33)$.

In our study caregivers in Southwestern Uganda receive emotional and instrumental support for their ailing patient with dementia from sources outside of the medical system These supports were most often obtained from one's religious community, social network, and one's own family. In a similar study in Ghana, Caregivers reported facing financial burden, social exclusion, emotional challenges , depression, and inadequate time for other social responsibilities and that the responsibilities around caregiving were mostly shared among close relatives(18). This is consistent with cultural values to care for one's family and community. Ideally, members of a lineage are expected to provide each other with instrumental and material resources and expect to receive emotional, economic, and social security. Though families offer a holistic approach to the needs of older people living with dementia, health and social policies offer inadequate scaffolding to support their work (34). With families becoming smaller, communities and society in general are important source of emotional and instrumental support for caregivers (35). In a study on Unmet Needs, Caregivers of patients who were referred to a dementia care program reported high levels of strain, low confidence in their ability to manage caregiving and access help, and frequently felt that they did not have a healthcare professional to help them with dementiarelated problems (36). The unique nature and prolonged duration of these needs directly influences the palliative care services and supports required by these family caregivers(37). The emotional and instrumental support for caregivers of patients with Alzheimer's disease and related dementia should entail resources like long term care insurance, provider care benefits, disability benefits for someone with dementia and social pensions as well as (1) physical, emotional, and psychological needs; (2) information and decisional support needs; and (3) instrumental support needs (38). Since acceptance, optimism, positive communication patterns, family connectedness, spirituality, social support, economic resources and the effective management of symptoms helps families adapt to the burdens of dementia care(39).

Most caregivers lack knowledge of the services or they perceive that services are too expensive. This is because care receivers present too many behavioural and emotional problems (40). Caregivers need to know that any unusual behavior in their aging family member is caused by disease, not by "craziness" or "meanness," and that they need new ways of responding to the person's cognitive abilities 
decline (41). In addition, caregivers need the right information, resources and training. The information needs to include understanding the characteristics and course of disease and what resources are available to the families along with training on how to care for people with dementia and how to prevent or deal with the patients' challenging behaviour (38). Health-care providers need to emphasize to caregivers of patients with ADRD the importance of maintaining their own physical and mental health so that they can continue to provide care for their loved one (42).

Several drawbacks were reported as a result of caregivers receiving support outside of the medical systems. Caregivers were told several conflicting messages that varied from individual to individual depending on the experience of whoever visits or shares with them. The individuals who offered this emotional and instrumental support were untrained and only relied on their lived experience or their social and spiritual expertise. The caregivers were faced with many conflicting messages that posed a big problem on which information or message to apply or not as they care for their ailing family member. This also runs the risk of misinformation when caregivers take medical advice from community members rather than trained medical professionals. Generally, family caregivers in low-income countries confront barriers in obtaining needed information about behavioral symptom management including but not limited to timely access to information, access to information that is tailored or specific to caregiver's needs and contexts and usable information that directly informs on how caregivers' manage behaviors(43).

The findings in our study therefore show that there is no psychosocial support given to Caregivers of patients with Alzheimer's disease in South-Western Uganda. Thus, greater resources for support need to be included within the medical system such as an extensive counseling package that is detailed and tailored towards the needs of the caregivers of patients with dementia that is designed and availed to all health workers at all levels of the health care system. A formal strong family and community social support system should also be encouraged to leverage and learn from the strategies employed by Caregivers as part of their lived experiences.

\section{Limitations Of The Study}

This was a qualitative study which provides in-depth insights into the experience of informal caregivers for patients with dementia in southwestern Uganda. As caregivers were recruited from hospitals with specialty psychiatry services, the findings of this study may 
not be generalized to caregivers of patients without access to these specialty diagnostic services

\section{Conclusions}

Caregivers of patients with Alzheimer's disease in southwestern Uganda receive unstructured emotional and instrumental supports from the community and family members based on individual experiences. The messages received from different sources are often conflicting, leaving the Caregivers in confusion. The medical care system offers some information about the clinical presentation of Alzheimer's disease but little or no psychosocial support to the Caregivers of patients with the disease. This has implications for how families and Caregivers cope with the day-to-day care of patients with Alzheimer's disease in Uganda and calls for institutionalization of structured psychosocial support for Caregivers to improve the care given to Alzheimer's patients in low-income countries.

\section{Declarations}

We declare no conflict of interest.

\section{Ethics approval and consent to participate}

Participants were interviewed after obtaining informed consent. Ethical Clearance was obtained from Mbarara University of Science and Technology-Research Ethics Committee (Approval Number: MUREC 1/7) and Uganda National Council of Science and Technology (Approval Number: SS4882). Permission was obtained from Hospital management and Psychiatry Unit heads of respective hospitals to access their records. Additionally, as participants often requested to be interviewed in their own home, local leaders of communities from which the participants resided were also engaged in a discussion of study procedure and risks/benefits and granted their approval of study procedures prior to data collection.

\section{Consent for publication}

Not applicable.

\section{Competing interests}

The authors declare that they have no competing interests. 


\section{Funding}

Research reported in this publication was supported by the Fogarty International Center and the National Institute on Aging of the National Institutes of Health under Award Number D43TW010128.

\section{Authors' contributions}

All authors contributed to the design of the study and writing of the manuscript. CA and ESO did the data analysis. GZR and CO provided scientific guidance throughout the project. All authors read and approved the final manuscript.

\section{Acknowledgements}

The primary author (CA) is a fellow under Mbarara Alzheimer's Disease Research Initiative (MADRI). We appreciate the expertise and assistance rendered by Prof. Elialilia S. Okello (ESO) during data analysis. We acknowledge the contribution by Dr. Christine Cooper-Vince (a mentor on the Mbarara Alzheimer's Disease Research Initiative) in the design of the study and writing of the manuscript. We recognize the great work done by Nabaasa Patience and Catherine Nakasiita, our research assistants for their dedication to the data collection and transcription.

\section{Authors' information}

CA is a Trainee on the on the Mbarara Alzheimer's Disease Research Initiative (MADRI) (Grant number D43TW010128: Celestino Obua). GZR is a Senior Lecturer in the Department of Psychiatry, Mbarara University of Science and Technology. He is also key person on the Mbarara Alzheimer's Disease Research Initiative (MADRI) - (Grant number D43TW010128: Celestino Obua). CO is a senior researcher, a Pharmacologist, and Vice Chancellor of Mbarara University of Science and Technology. He is the Principal Investigator Grant number D43TW010128: Celestino Obua). EW is a Research Administrator/Manager in the Office of Research Administration at Mbarara University of Science and Technology. She is also key person on the Mbarara Alzheimer's Disease Research Initiative (MADRI) (Grant number D43TW010128: Celestino Obua).

\section{References}

1. Alzheimer's A. 2013 Alzheimer's disease facts and figures. Alzheimers Dement. 2013;9(2):208-45. 
2. Prince M, Bryce R, Albanese E, Wimo A, Ribeiro W, Ferri CP. The global prevalence of dementia: a systematic review and metaanalysis. Alzheimers Dement. 2013;9(1):63-75 e2.

3. ADI. Dementia in sub-Saharan Africa: Challenges and opportunities. Illinois, USA: 2017.

4. Prince M, Bryce R, Albanese E, Wimo A, Ribeiro W, Ferri CP. The global prevalence of dementia: a systematic review and metaanalysis. Alzheimer's \& dementia. 2013;9(1):63-75. e2.

5. Malhotra P. Impairments of Attention in Alzheimer's Disease. Current opinion in psychology. 2018.

6. Cheng ST. Dementia Caregiver Burden: a Research Update and Critical Analysis. Curr Psychiatry Rep. 2017;19(9):64.

7. Chiao $\mathrm{C}-\mathrm{Y}$, Wu H-S, Hsiao C-Y. Caregiver burden for informal caregivers of patients with dementia: A systematic review. International Nursing Review. 2015;62:340-50.

8. Schneider J, Murray J, Banerjee S, Mann A. EUROCARE: a cross-national study of co-resident spouse carers for people with Alzheimer's disease: I-factors associated with carer burden. International journal of geriatric psychiatry. 1999;14(8):651-61.

9. Schulz R, Martire LM. Family Caregiving of Persons With Dementia: Prevalence, Health Effects, and Support Strategies. The American Journal of Geriatric Psychiatry. 2004;12(3):240-9.

10. Schulz R, O'Brien AT, Bookwala J, Fleissner K. Psychiatric and physical morbidity effects of dementia caregiving: prevalence, correlates, and causes. Gerontologist. 1995;35(6):771-91.

11. Allen AP, Curran EA, Duggan A, Cryan JF, Chorcorain AN, Dinan TG, et al. A systematic review of the psychobiological burden of informal caregiving for patients with dementia: Focus on cognitive and biological markers of chronic stress. Neurosci Biobehav Rev. 2017;73:123-64.

12. Kales HC, Gitlin LN, Lyketsos CG. Assessment and management of behavioral and psychological symptoms of dementia. Bmj. 2015;350:h369.

13. Sinha P, Desai NG, Prakash O, Kushwaha S, Tripathi CB. Caregiver burden in Alzheimer-type dementia and psychosis: A comparative study from India. Asian Journal of Psychiatry. 2017;26:86-91.

14. Acosta D, Rottbeck R, Rodriguez G, Ferri CP, Prince MJ. The epidemiology of dependency among urban-dwelling older people in the Dominican Republic; a cross-sectional survey. BMC Public Health. 2008;8:285.

15. Liu Z, Albanese E, Li S, Huang Y, Ferri CP, Yan F, et al. Chronic disease prevalence and care among the elderly in urban and rural Beijing, China - a 10/66 Dementia Research Group cross-sectional survey. BMC Public Health. 2009;9:394.

16. Raggi A, Tasca D, Panerai S, Neri W, Ferri R. The burden of distress and related coping processes in family caregivers of patients with Alzheimer's disease living in the community. Journal of the neurological sciences. 2015;358(1-2):77-81.

17. Wang J, Xiao LD, He G-P, Ullah S, De Bellis A. Factors contributing to caregiver burden in dementia in a country without formal caregiver support. Aging \& mental health. 2014;18(8):986-96.

18. Ae-Ngibise KA, Doku VCK, Asante KP, Owusu-Agyei S. The experience of caregivers of people living with serious mental disorders: a study from rural Ghana. Global health action. 2015;8(1):26957. 
19. Ayalew M, Workicho A, Tesfaye E, Hailesilasie H, Abera M. Burden among caregivers of people with mental illness at Jimma University Medical Center, Southwest Ethiopia: a cross-sectional study. Annals of General Psychiatry. 2019;18(1):10.

20. Selwood A, Johnston K, Katona C, Lyketsos C, Livingston G. Systematic review of the effect of psychological interventions on family caregivers of people with dementia. Journal of affective disorders. 2007;101(1-3):75-89.

21. Brodaty $\mathrm{H}$, Arasaratnam $\mathrm{C}$. Meta-analysis of nonpharmacological interventions for neuropsychiatric symptoms of dementia. Am J Psychiatry. 2012;169(9):946-53.

22. Gavrilova SI, Ferri CP, Mikhaylova N, Sokolova O, Banerjee S, Prince M. Helping carers to care-the $10 / 66$ dementia research group's randomized control trial of a caregiver intervention in Russia. Int J Geriatr Psychiatry. 2009;24(4):347-54.

23. Dias A, Dewey ME, D'Souza J, Dhume R, Motghare DD, Shaji KS, et al. The effectiveness of a home care program for supporting caregivers of persons with dementia in developing countries: a randomised controlled trial from Goa, India. PLoS One. 2008;3(6):e2333.

24. Anderson JG, Hundt E, Rose KM. Nonpharmacological Strategies Used By Family Caregivers of Persons With Alzheimer's Disease and Related Dementias as Presented in Blogs. Journal of Gerontological Nursing. 2019;45(7):25-35.

25. Roberto KA, Blieszner R. Diverse family structures and the care of older persons. Canadian Journal on Aging/La Revue canadienne du vieillissement. 2015;34(3):305-20.

26. Uganda Bureau of Statistics U. National population and housing census 2014. Uganda Bureau of Statistics Kampala (Uganda); 2014.

27. Braun V, Clarke V. Using thematic analysis in psychology. Qualitative research in psychology. 2006;3(2):77-101.

28. Muhr T. Atlas. ti: qualitative data analysis, version 7. Berlin: Scientific Software Development GmbH. 2013.

29. Thrush A, Hyder A. The neglected burden of caregiving in low-and middle-income countries. Disability and health journal. 2014;7(3):262-72.

30. Agyeman N, Guerchet M, Nyame S, Tawiah C, Owusu-Agyei S, Prince MJ, et al. "When someone becomes old then every part of the body too becomes old": Experiences of living with dementia in Kintampo, rural Ghana. Transcult Psychiatry. 2019:1363461519847054.

31. Deist M, Greeff AP. Living with a parent with dementia: A family resilience study. Dementia (London). 2017;16(1):126-41.

32. Hindley G, Kissima J, L LO, Paddick SM, Kisoli A, Brandsma C, et al. The role of traditional and faith healers in the treatment of dementia in Tanzania and the potential for collaboration with allopathic healthcare services. Age Ageing. 2017;46(1):130-7.

33. Piersol CV, Canton K, Connor SE, Giller I, Lipman S, Sager S. Effectiveness of interventions for caregivers of people with Alzheimer's disease and related major neurocognitive disorders: A systematic review. American Journal of Occupational Therapy. 2017;71(5):7105180020p1-p10. 
34. Agyeman N, Guerchet M, Nyame S, Tawiah C, Owusu-Agyei S, Prince MJ, et al. "When someone becomes old then every part of the body too becomes old": Experiences of living with dementia in Kintampo, rural Ghana. Transcultural psychiatry. 2019:1363461519847054.

35. Nishio M, Kimura H, Ogomori K, Ogata K. Emotional and instrumental support influencing male caregivers for people with dementia living at home. Journal of Rural Medicine. 2017;12(1):20-7.

36. Jennings LA, Reuben DB, Evertson LC, Serrano KS, Ercoli L, Tan Z, et al., editors. UNMET NEEDS OF CAREGIVERS OF PATIENTS REFERRED TO A DEMENTIA CARE PROGRAM. JOURNAL OF GENERAL INTERNAL MEDICINE; 2013: SPRINGER 233 SPRING ST, NEW YORK, NY 10013 USA.

37. Thompson GN, Roger K. Understanding the needs of family caregivers of older adults dying with dementia. Palliative \& supportive care. 2014;12(3):223-31.

38. WHO WHO. Supporting Informal Caregivers of People Living with Dementia. Interantional AsD; 2015 2015. Report No.

39. Deist M, Greeff AP. Living with a parent with dementia: A family resilience study. Dementia. 2017;16(1):126-41.

40. Huang HL, Shyu YIL, Chen ST, Hsu WC. Caregiver self-efficacy for managing behavioural problems of older people with dementia in Taiwan correlates with care receivers' behavioural problems. Journal of Clinical Nursing. 2009;18(18):2588-95.

41. Musisi S. Alzheimer's Disease Harvard University: Harvard Health; 2018.

42. Richardson TJ, Lee SJ, Berg-Weger M, Grossberg GT. Caregiver health: health of caregivers of Alzheimer's and other dementia patients. Current psychiatry reports. 2013;15(7):367.

43. Werner NE, Stanislawski B, Marx KA, Watkins DC, Kobayashi M, Kales H, et al. Getting what they need when they need it. Applied clinical informatics. 2017;26(01):191-205.

\section{Supplementary Files}

This is a list of supplementary files associated with this preprint. Click to download.

- Studyparticipants.docx 